\title{
Divalent cations as probes for structure-function relationships of cloned voltage-dependent sodium channels
}

\author{
M. Pusch* \\ Max-Planck-Institut für biophysikalische Chemie, Am Fassberg, D-3400 Göttingen, Federal Republic of Germany
}

Received March 14, 1990/Accepted in revised form May 23, 1990

\begin{abstract}
Several cloned sodium channels were expressed in oocytes and compared with respect to their sensitivity to internal $\mathrm{Mg}^{2+}$ concerning the open-channel block and to external $\mathrm{Ca}^{2+}$ concerning open-channel block and shifts in steady-state activation. 2. A quantitative comparison between wild-type II channels and a mutant with a positive charge in the $\mathrm{S} 4$ segment of repeat I neutralized (K226Q) revealed no significant differences in the $\mathrm{Mg}^{2+}$ block. 3. The blocking effect of extracellular $\mathrm{Ca}^{2+}$ ions on single-channel inward currents was studied for type II, mutant $\mathrm{K} 226 \mathrm{Q}$ and type III. A quantitative comparison showed that all three channel types differ significantly in their $\mathrm{Ca}^{2+}$ sensitivity. 4 . The influence of extracellular $\mathrm{Ca}^{2+}$ on the voltage dependence of steadystate activation of macroscopic currents was compared for type II and K226Q channels. Extracellular $\mathrm{Ca}^{2+}$ increases the voltage of half-maximal activation, $V_{1 / 2}$, more for K226Q than for wild-type II channels; a plot of $V_{1 / 2}$ against $[\mathrm{Ca}]_{o}$ is twice as steep for the mutant $\mathrm{K} 226 \mathrm{Q}$ as for the wild-type on a logarithmic concentration scale. 5. The differential effects of extracellular $\mathrm{Ca}^{2+}$ and intracellular $\mathrm{Mg}^{2+}$ on wild-type II and K226Q channels are discussed in terms of structural models of the $\mathrm{Na}^{+}$channel protein.
\end{abstract}

Key words: Sodium channel - Divalent cations $-\mathrm{Mg}^{2+}$ block - Patch clamp - Transmembrane topology

\section{Introduction}

The previous paper (Pusch 1990) demonstrates that internal $\mathrm{Mg}^{2+}$ produces an open-channel block in several cloned $\mathrm{Na}^{+}$channels. The action of extracellular divalent cations on ion permeation through voltage-dependent $\mathrm{Na}^{+}$channels in neuronal (Woodhull 1973; Taylor et al.

Abbreviations: $[\mathrm{Na}]_{i}$, intracellular $\mathrm{Na}^{+}$concentration; $[\mathrm{Mg}]_{i}$, intracellular $\mathrm{Mg}^{2+}$ concentration; $[\mathrm{Ca}]_{0}$, extracellular $\mathrm{Ca}^{2+}$ concentration

Present address and address for offprint requests: Istituto di Cibernetica e Biofisica, CNR, Via Dodecaneso 33, I-16146 Genova, Italy
1976; Yamamoto et al. 1984; Worley et al. 1986; Vandenberg and Bezanilla 1988) and cardiac (Nilius 1988) cells has been investigated at macroscopic and single-channel levels. It has been shown that external $\mathrm{Ca}^{2+}$ ions exert a voltage-dependent blocking effect and that they probably share at least one common binding site with tetrodotoxin and saxitoxin. Thus divalent cations at either side of the membrane interfere with sodium permeation through voltage-dependent $\mathrm{Na}^{+}$channels. In addition to the blocking effect, elevated $[\mathrm{Ca}]_{o}$ shifts steady-state activation curves to more positive voltages (Frankenhaeuser and Hodgkin 1957; Hille et al. 1975; Hahin and Campbell 1983).

The successful expression of cloned $\mathrm{Na}^{+}$channels in Xenopus oocytes (Stühmer et al. 1987) makes it possible to compare functional properties of genetically different $\mathrm{Na}^{+}$channels. Stühmer et al. (1989) examined activation and inactivation properties in several mutants of ratbrain type II $\mathrm{Na}^{+}$channel expressed in Xenopus oocytes. The present paper investigates the effects of intracellular $\mathrm{Mg}^{2+}$ and extracellular $\mathrm{Ca}^{2+}$ on rat brain type II, type III and a mutant of the type II channel.

In the mutant "K226Q" the positive amino acid lysine at homologous position 226 of th type II sodium channel (Noda et al. 1986) is replaced by an uncharged glutamine. Position 226 is located at the C-terminal end of the 'S4 segment' of homologous repeat I. This segment has been shown to be involved in the voltage-dependent activation of the $\mathrm{Na}^{+}$channel (Stühmer et al. 1989); recent models of the membrane topology of the $\mathrm{Na}^{+}$channel predict that position 226 is close to the intracellular side of the membrane (Noda et al. 1986; Guy 1988). The different sensitivities of wild-type and mutated channels to intra- and extracellular divalent ions may provide additional structural information about the topology of the sodium channel.

\section{Methods}

The methods are identical to those of the companion paper (Pusch 1990). 


\section{Results}

\section{1. $\mathrm{Mg}^{2+}$ block in wild-type II and $\mathrm{K} 226 \mathrm{Q}$ channels}

Intracellular $\mathrm{Mg}^{2+}$ blocks outward currents in the mutant $\mathrm{K} 226 \mathrm{Q}$ in a voltage-dependent manner, similar to that seen in wild-type II channels (Pusch 1990). For a quantitative comparison of the $\mathrm{Mg}^{2+}$ block between wild-type II and K226Q channels, macroscopic tail-currents through K226Q channels were measured in large inside-out patches. In Fig. 1 tail-current I-V's at zero and $5 \mathrm{mM}[\mathrm{Mg}]_{i}$ and two different $[\mathrm{Na}]_{i}$ 's are shown. These I-V's look similar to those from the wild-type (Fig. 6, companion paper, Pusch (1990)). Several I-V's were fitted according to the procedure described previously (Pusch $1990)$ to obtain the half-maximal blocking concentration $K_{d}(0)$ at $V=0$ and the electrical distance $\delta$ (see legend of Fig. 1). The parameters obtained from the fit are listed in Table 1. For comparison, the corresponding parameters obtained for the wild-type are also included. The solid lines in Fig. 1 represent the fit. The dashed lines at $5 \mathrm{mM}$ $[\mathrm{Mg}]_{i}$ correspond to the fit parameters for the wild-type. It can be seen that at both $\mathrm{Na}^{+}$concentrations the $\mathrm{Mg}^{2+}$ block is quantitatively similar in the mutant K226Q and the wild-type.

At $30 \mathrm{~m} M[\mathrm{Na}]_{i}$ the parameters $K_{d}(0)$ and $\delta$ are nearly the same as those for the wild-type. At $125 \mathrm{mM}[\mathrm{Na}]_{i}$, the half-maximal blocking concentration at $V=0$ is about half the value of that for wild-type. However, this is not a significant difference, as the deviations of the fits from the data points are larger than the difference between the fits (see Fig. $1 \mathrm{~B}, 5 \mathrm{mM} \mathrm{Mg}^{2+}$ ).

In addition to the similarity in the $\mathrm{Mg}^{2+}$ block, the selectivity (data not shown) and the single-channel conductance (see below) for the mutant K226Q are not significantly different from those of the wild-type.

The analysis of current-voltage relationships for the type III channel (see Table 1) shows that it does not differ dramatically in its $\mathrm{Mg}^{2+}$ sensitivity from the type II channel.

\section{Extracellular $\mathrm{Ca}^{2+}$ block of single-channel currents}

Voltage-dependent block of $\mathrm{Na}^{+}$inward currents by extracellular $\mathrm{Ca}^{2+}$ ions has been reported for several preparations (Taylor et al. 1976; Yamamoto et al. 1984; Nilius 1988; Tanguy and Yeh 1988). Here, the $\mathrm{Ca}^{2+}$ block is used to compare different genetically defined $\mathrm{Na}^{+}$ channel types.

Figure 2 shows representative single-channel current traces from inside-out patches of oocytes injected with type II, K226Q and type III mRNA at different voltages with 0 or $4 \mathrm{mM} \mathrm{Ca}{ }^{2+}$ in the pipette solution. Apart from the longer mean open time characteristic for type III channels, a reduction of the open-channel current amplitude at $4 \mathrm{mM} \mathrm{Ca}^{2+}$ compared to $0 \mathrm{Ca}^{2+}$ for all channel types is discernible.

Single-channel current amplitudes were analysed as previously described (Pusch 1990). Figure 3 shows the averaged amplitudes for the different channel types versus

\section{Mutant K226Q}

A $30 \mathrm{Na}$
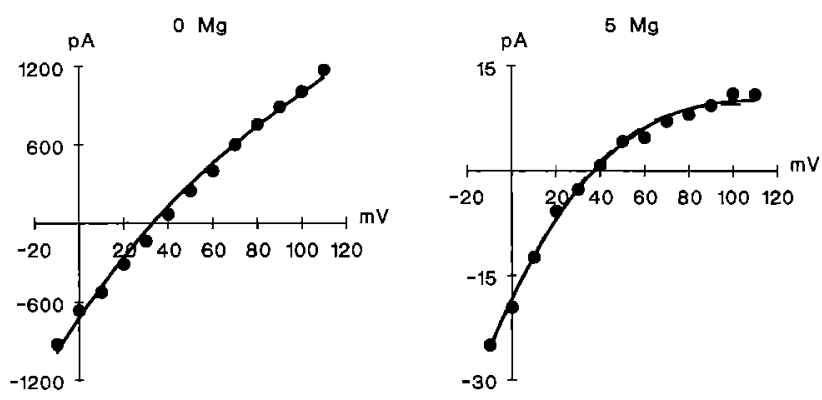

B $125 \mathrm{Na}$
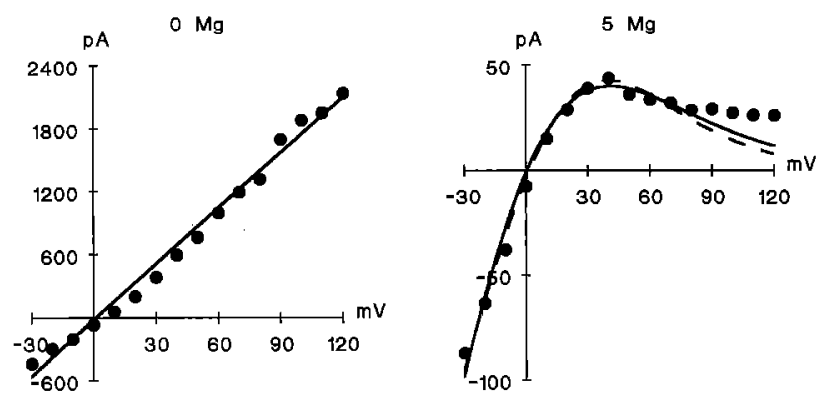

Fig. 1 A, B. Instantaneous current-voltage relationships for mutant K226Q. The I-V's were derived from the tail currents measured with large inside-out patches as described in Pusch (1990). The pipette solution was in all cases normal frog Ringer (see Pusch 1990); the bath solution contained in $\mathrm{A}$ (in $\mathrm{mM}$ ): $30 \mathrm{NaCl}, 90 \mathrm{KCl}, 10 \mathrm{KOH}$ HEPES, $10 \mathrm{KOH}$-EGTA, pH 7.2; in B: $105 \mathrm{NaCl}, 10 \mathrm{NaOH}-$ HEPES, $10 \mathrm{NaOH}$-EGTA, $2.5 \mathrm{KCl}, \mathrm{pH} 7.2 .[\mathrm{Mg}]_{i}$ is given in $\mathrm{m} M$. The solid lines represent fits of the following equation which is identical to (1) of the companion paper (Pusch 1990, see there for the meaning of the symbols):

$I=P \frac{V F^{2}}{R T} \frac{1-\exp \left(-\frac{F\left(V-V_{r}\right)}{R T}\right)}{1-\exp \left(-\frac{F V}{R T}\right)} \frac{1}{1+\frac{[\mathrm{Mg}]}{K_{d}(0)} \exp \left(\frac{\delta z F V}{R T}\right)}$.

The essential parameters, $K_{d}(0)$ and $\delta$, are listed in Table 1. Dashed lines at $[\mathrm{Mg}]_{i}=5 \mathrm{mM}$ were obtained with the $\mathrm{Mg}^{2+}$ block parameters of wild-type II (see Table 1)

Table 1. $\mathrm{Mg}^{2+}$ block parameters. Parameters describing the $\mathrm{Mg}^{2+}$ block obtained by fitting a simple voltage-dependent block model (see (1) in Pusch (1990)) to several I-V's for different channel types. $K_{d}(0)$ denotes the half-maximal blocking concentration at $V=0, \delta$ the electrical distance. The error estimates were obtained as described previously (Pusch 1990). The values for type II were taken from Table 1 of the companion paper (Pusch 1990)

\begin{tabular}{rlrll}
\hline \begin{tabular}{l}
$\begin{array}{l}{[\mathrm{Na}]_{i}} \\
\text { in } M\end{array}$ \\
\hline 30
\end{tabular} & Channel & \multicolumn{1}{c}{$\begin{array}{l}K_{d}(0) \\
\text { in } \mathrm{m} M\end{array}$} & $\delta$ & $\begin{array}{l}\text { Number } \\
\text { of I-V's }\end{array}$ \\
& K226Q & $18.1 \pm 2.3$ & $0.22 \pm 0.02$ & 16 \\
$\approx 125$ & Type II & $21.5 \pm 4.7$ & $0.25 \pm 0.02$ & 37 \\
& K226Q & $5.7 \pm 1.1$ & $0.40 \pm 0.02$ & 12 \\
\multirow{2}{*}{30} & Type II & $13.0 \pm 2.2$ & $0.50 \pm 0.02$ & 8 \\
& Type III & 7.5 & 0.25 & 6 \\
\hline
\end{tabular}


A

Type II

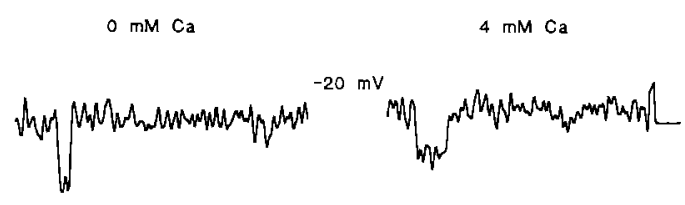

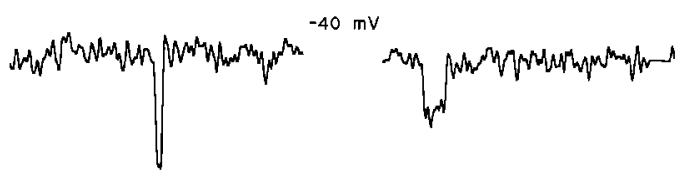

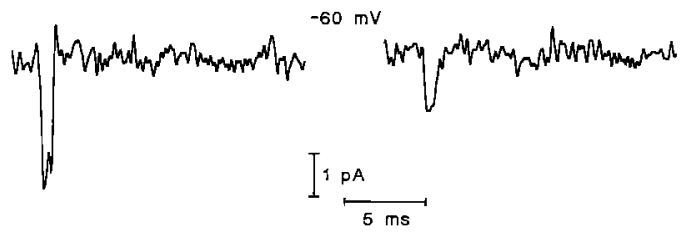

B Mutant K226Q

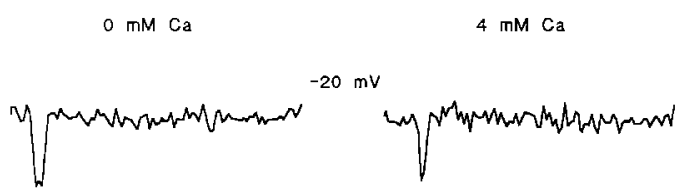

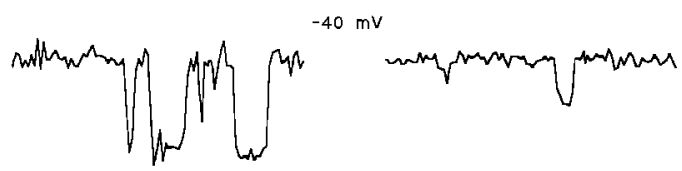

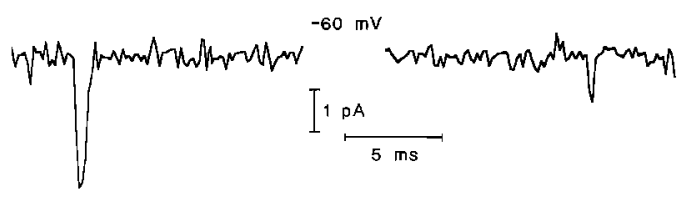

C

Type III
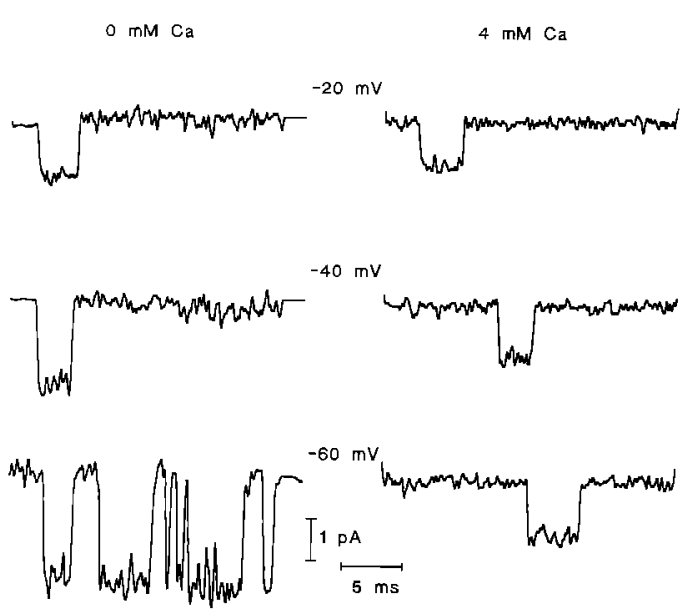

Fig. 2. Single-channel current traces for wild-type II (A), K226Q (B) and type III (C) channels measured on inside-out patches at the indicated voltages. Bath solution (in $\mathrm{mM}$ ): $90 \mathrm{KCl}, 30 \mathrm{NaCl}, 10$ KOH-HEPES, $10 \mathrm{KOH}-\mathrm{EGTA}$, pH 7.2. Pipette solution: 115 $\mathrm{NaCl}, 10 \mathrm{NaOH}$-HEPES, $2.5 \mathrm{KCl}, \mathrm{pH} 7.2$ and $4 \mathrm{CaCl}_{2}$ for the right current traces. Currents were filtered at $4 \mathrm{kHz}$

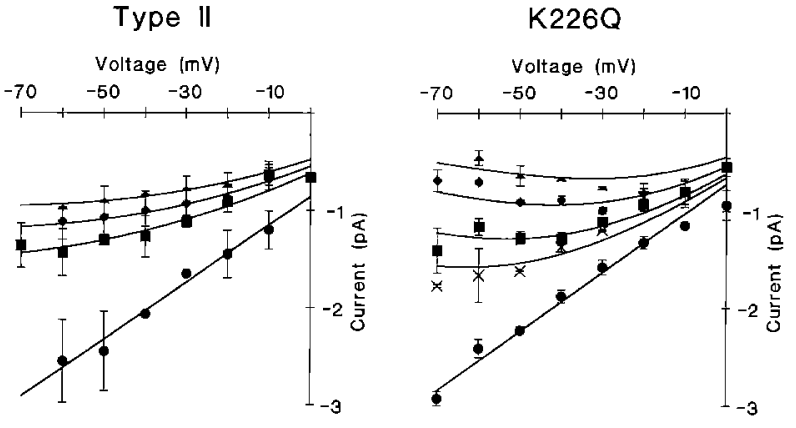

Type III

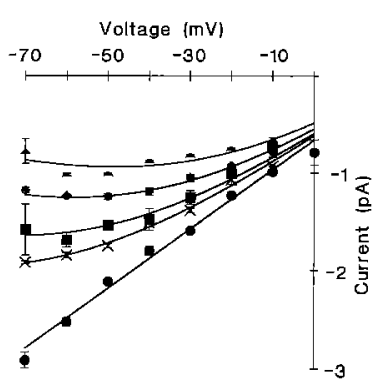

Fig. 3. Single-channel amplitudes for wild-type II, K226Q and type III channels versus test voltage at different extracellular $\mathrm{Ca}^{2+}$ concentrations. Each data point is the mean of at least two values obtained from different patches. The solutions are as in Fig. 2 with the following $\mathrm{CaCl}_{2}$ concentrations in the pipette (in $\mathrm{m} M$ ): circles: 0 ; crosses: 1 ; squares: 1.8 ; diamonds: 4 ; triangles: 8.3 . The solid lines represent fits of (2) with the parameters listed in Table 2

test voltage at different $\mathrm{Ca}^{2+}$ concentrations. Qualitatively it can already be seen that the $\mathrm{Ca}^{2+}$ dependence of the current-voltage relationships for type II and the mutant $\mathrm{K} 226 \mathrm{Q}$ is markedly different, while it appears similar for type III and K226Q channels.

In order to obtain a quantitative analysis of the block, the dependence of the single-channel current $i$ on membrane voltage $V$ and $\mathrm{Ca}^{2+}$ concentration $c$ was determined using the following equation:

$i=\frac{\gamma\left(V-V_{r}\right)}{1+\frac{c}{K_{d}(0)} \exp \left(\frac{-2 \delta V F}{R T}\right)}$.

The simple Ohmic conduction law instead of the Goldman-Hodgkin-Katz (GHK) current equation was applied to describe the $\mathrm{I}-\mathrm{V}$ at zero $\mathrm{Ca}^{2+}$ because of the linearity of both the GHK current equation at voltages below the reversal potential and the experimental I-V's at zero $\mathrm{Ca}^{2+}$ (see Fig. 3). In (1) $\gamma$ denotes the single-channel conductance, $V_{r}$ the reversal potential, $K_{d}(0)$ the half-maximal block concentration at $V=0$ and $\delta$ the electrical distance of the $\mathrm{Ca}^{2+}$ binding site from the outside. Underlying this relation is the assumption of a simple bimolecular voltage-dependent block (Hill coefficient $=1$ ). However, the concentration dependence of currentvoltage relationships of the wild-type II channel could not be described by (1). In order to allow for more complicated block mechanisms, a Hill coefficient different from 
Table 2. $\mathrm{Ca}^{2+}$ block parameters. Parameters describing the $\mathrm{Ca}^{2+}$ block for different channel types obtained by fitting (2) to all measured single-channel amplitudes. $K_{d}(0)$ denotes the half-maximal blocking concentration at $V=0, \delta$ the electrical distance, $n$ the Hill coefficient, $\gamma$ the single-channel conductance in $0 \mathrm{mM} \mathrm{Ca}^{2+}$, and $V_{r}$ the reversal potential. The error estimates were obtained as described in Pusch (1990)

\begin{tabular}{llllll}
\hline Channel & $\begin{array}{l}K_{d}(0) \\
\text { in } \mathrm{m} M\end{array}$ & $\delta$ & $n$ & $\begin{array}{l}\gamma \\
\text { in pS }\end{array}$ & $\begin{array}{l}V_{r} \\
\text { in } \mathrm{mV}\end{array}$ \\
\hline Type II & $13.7 \pm 5.8$ & $0.17 \pm 0.04$ & $0.46 \pm 0.05$ & $29.4 \pm 1.6$ & $29.3 \pm 3.5$ \\
K226Q & $14.8 \pm 3.6$ & $0.36 \pm 0.05$ & $0.83 \pm 0.07$ & $30.3 \pm 1.0$ & $23.9 \pm 2.3$ \\
Type III & $31.2 \pm 6.8$ & $0.32 \pm 0.04$ & $0.75 \pm 0.05$ & $30.5 \pm 1.0$ & $21.7 \pm 2.0$ \\
\hline
\end{tabular}

1 was admitted:

$$
i=\frac{\gamma\left(V-V_{r}\right)}{1+\left(\frac{c}{K_{d}(0)}\right)^{n} \exp \left(\frac{-2 \delta V F}{R T}\right)} .
$$

This equation provided a satisfactory description of all measured I-V's. A fit of (2) to the data points shown in Fig. 3 yielded the parameters listed in Table 2; the solid lines in Fig. 3 represent the fit. Th single-channel conductance is nearly identical in all channel types. However, in zero $\mathrm{Ca}^{2+}$ it is approximately twice as large as that reported by Stühmer et al. (1989) for the type II channel. This discrepancy can be explained by their use of a pipette solution containing $1.8 \mathrm{mM} \mathrm{Ca}{ }^{2+}$, which, as can be seen in Fig. 3, decreases the steepness of the single-channel I-V significantly and introduces curvature.

The relatively low value of the Hill coefficient obtained for the wild-type II $(n=0.46)$ means that the binding mechanism of $\mathrm{Ca}^{2+}$ is characterized by negative cooperativity. The substantial differences in the Hill coefficient and the electrical distance for wild-type II and K226Q channels ( $n=0.46, \delta=0.17$ for wild-type II; $n=0.83, \delta=0.36$ for $\mathrm{K} 226 \mathrm{Q}$ ) are noteworthy since the mutant K226Q differs from wild-type only by the neutralization of a positive amino acid (lysine) which is thought to be located close to the intracellular side of the membrane. The concentration for half-maximal $\mathrm{Ca}^{2+}$ block at $V=0$ is virtually identical in both channel types $\left(K_{d}(0) \approx\right.$ $14 \mathrm{~m} M)$.

$K_{d}(0)$ is twice as large for the type III $\mathrm{Na}^{+}$channel as compared to the wild-type II and the type II mutant $\mathrm{K} 226 \mathrm{Q}$, whereas the values of the electrical distance $\delta$ and the Hill coefficient $n$ are similar to those of the mutant K226Q. However, since the primary sequence of the type III $\mathrm{Na}^{+}$channel differs from that of the type II at many positions (Kayano et al. 1988), the results for the type III channel are difficult to interpret in structural terms.

\section{Comparison of the shift of activation by external $\mathrm{Ca}^{2+}$ between wild-type II and $K 226 Q$ channels}

Steady-state activation curves of $\mathrm{Na}^{+}$currents are shifted to more positive voltages at higher external divalent cation concentrations (Frankenhaeuser and Hodgkin 1957; Hille et al. 1975; Hahin and Campbell 1983). To investigate and compare further functional properties of wild-type II and K226Q channels, the effect of external $\mathrm{Ca}^{2+}$ on steady-state activation was examined for both channel types.

Figure $4 \mathrm{~A}$ shows representative current traces from inside-out patches at 0 and $8.3 \mathrm{mM} \mathrm{Ca}{ }^{2+}$ in the pipette solution for type II and K226Q channels. In order to investigate the effect of $\mathrm{Ca}^{2+}$ on gating without interference by the voltage-dependent open-channel block, the currents at non-zero $[\mathrm{Ca}]_{o}$ were scaled by the factor

$1+\left([\mathrm{Ca}]_{o} / K_{d}(0)\right)^{n} \exp (-2 \delta V F / R T)$,

with the parameters obtained from the single-channel measurements described above (see Table 2). The scaled current traces are shown as dashed lines in Fig. 4A.

Comparing the current traces at $0 \mathrm{Ca}^{2+}$ shows that their is little difference in the degree of activation for the two channel types. However, there was also some variation in the steady-state activation properties from patch to patch. For both channel types a comparison of the current traces at 0 and $8.3 \mathrm{mM} \mathrm{Ca}{ }^{2+}$ shows that $\mathrm{Ca}^{2+}$ has a dramatic effect on the degree of activation at different voltages.

To quantify activation, the following equation was fitted to peak current-voltage relationships:

$$
\begin{aligned}
I= & P \frac{V F^{2}}{R T} \frac{1-\exp \left(\frac{-\left(V-V_{r}\right) F}{R T}\right)}{1-\exp \left(\frac{-V F}{R T}\right)} \\
& \cdot \frac{1}{\left(1+\exp \left(\frac{z_{m} F\left(V_{1 / 2}-V\right)}{R T}\right)\right)^{3}} .
\end{aligned}
$$

The first part is the Goldman-Hodgkin-Katz current equation. The second part describes steady-state activation $m_{\infty}$ :

$m_{\infty}=\frac{1}{1+\exp \left(\frac{z_{m} F\left(V_{1 / 2}-V\right)}{R T}\right)}$,

with the voltage of half-maximal activation, $V_{1 / 2}$, and the apparent gating valence, $z_{m}$. The factor $m_{\infty}^{3}$ in (3) is based on the assumption of three independent identical activation 'particles' according to the Hodgkin-Huxley-model (Hodgkin and Huxley 1952). This model serves as a quantitative description of the experiments; no special mechanistic significance is implied.

Equation (3) effectively describes all peak currentvoltage relationships. Figure 4B shows the $m_{\infty}^{3}$ curves for the results obtained in Fig. 4A. Circles represent wildtype, squares mutant K226Q, filled symbols correspond to $0 \mathrm{Ca}^{2+}$ and open symbols to $8.3 \mathrm{mMCa}^{2+}$. It is evident that for both channel types the activation curves are shifted to the right; $m_{\infty}^{3}$ curves seem to be steeper for the wild-type than for the mutant channels, indicating that less charge movement is associated with activation of 
A

a
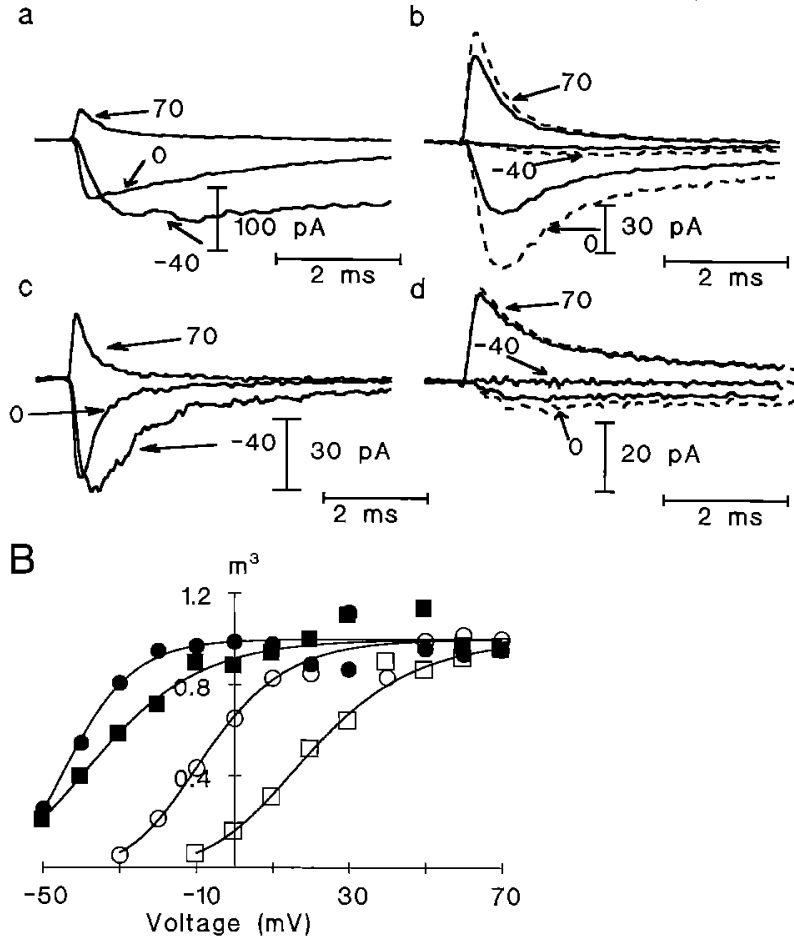

Fig. 4. A Representative current traces in 0 (a and $\mathbf{c}$ ) and 8.3 (b and d) $\mathrm{mM} \mathrm{Ca}{ }^{2+}$ from different inside-out patches for type II (a and b) and K226Q (c and d) channels. Starting from a holding voltage of $\approx-100 \mathrm{mV}$, steps to the indicated voltages were applied. Each current trace is the mean of 8 individual recordings. Linear leakage and capacitive currents were subtracted using a $\mathbf{P} / 4$ method. The solutions were the same as in Fig. 2 with no added $\mathrm{CaCl}_{2}$ in the pipette for $\mathbf{a}$ and $\mathbf{c}$ and $8.3 \mathrm{mM} \mathrm{CaCl}$ for $\mathbf{b}$ and $\mathbf{d}$. The dashed lines were obtained from the original traces by scaling with the factor:

$1+\left(8.3 \mathrm{mM} / K_{d}(0)\right)^{n} \exp (-2 \delta V F / R T)$

with the values for $K_{d}(0), n$ and $\delta$ taken from Table 2 (see text). B Steady-state activation $m_{\infty}^{3}$ versus test voltage for the currents of the experiments which are illustrated in $\mathbf{A}$ (in $\mathbf{A}$, for clarity, not all traces are shown). Circles: type II; squares: mutant K226Q; filled symbols: $0 \mathrm{Ca}^{2+}$; open symbols: $8.3 \mathrm{mM} \mathrm{Ca}{ }^{2+}$. The solid lines correspond to (3) and (4) with the following parameters: Type II,

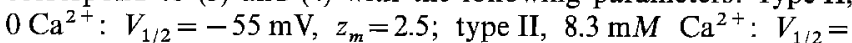
$-25 \mathrm{mV}, z_{m}=1.9 ; \mathrm{K} 226 \mathrm{Q}, 0 \mathrm{Ca}^{2+}: V_{1 / 2}=-55 \mathrm{mV}, z_{m}=1.6 ; \mathrm{K} 226 \mathrm{Q}$, $8.3 \mathrm{mMCa} \mathrm{Ca}^{2+}: V_{1 / 2}=-3 \mathrm{mV}, z_{m}=1.5$

K226Q channels than with activation of wild-type II channels.

In Fig. 5 the mean values of $V_{1 / 2}$ and $z_{m}$ obtained from several experiments are plotted versus $[\mathrm{Ca}]_{a}$. The degree of shift of $V_{1 / 2}$ with [Ca] $]_{o}$ is larger for the mutant than for the wild-type (Fig. 5A). The solid lines in Fig. 5A represent fits of an exponential function of the form

$V_{1 / 2}=V_{\infty}-\left(V_{\infty}-V_{0}\right) \exp \left(-[\mathrm{Ca}]_{o} / K\right)$

with

$V_{\infty}=-29 \mathrm{mV}, \quad V_{0}=-48 \mathrm{mV}, \quad K=5.9 \mathrm{mM}$

for wild-type II and

$V_{\infty}=-6 \mathrm{mV}, \quad V_{0}=-43 \mathrm{mV}, \quad K=3.0 \mathrm{mM}$
A mv
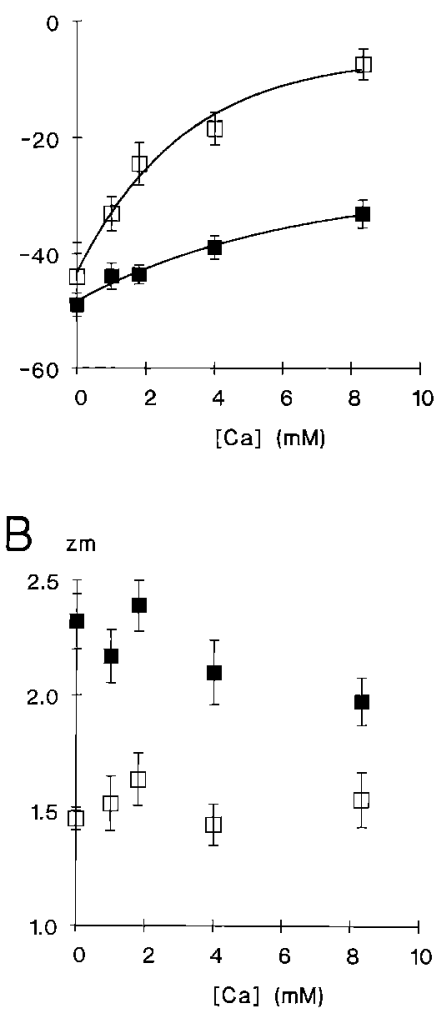

Fig. 5. Mean values of voltage for half-maximal activation, $V_{1 / 2}$, (A) and of the apparent gating valence, $z_{m}$, (B) for wild-type II (filled symbols) and mutant $\mathrm{K} 226 \mathrm{Q}$ (open symbols) versus $[\mathrm{Ca}]_{0}$. Error bars represent statistical standard deviations. Solid lines in $\mathbf{A}$ are fits of an exponential function (5) with the parameters given in the text

for mutant K226Q. The values for $K$ denote that $V_{1 / 2}-V_{\infty}$ changes e-fold per $6 \mathrm{mM}$ change in $[\mathrm{Ca}]_{0}$ for the wild-type, whereas only $3 \mathrm{mM} \mathrm{Ca}{ }^{2+}$ is needed for an e-fold change in the mutant. The steady-state activation shifts indicate that the mutant K226Q has a significantly higher sensitivity to extracellular $\mathrm{Ca}^{2+}$ than the wildtype.

The value of $z_{m}$ does not strongly depend on $[\mathrm{Ca}]_{o}$ as can be seen in Fig. 5B. However, $z_{m}$ decreases slightly with increasing $[\mathrm{Ca}]_{0}$ for the wild-type, whereas it is relatively constant for K226Q channels. The absolute value of $z_{m}$ is lower for the mutant at all $\mathrm{Ca}^{2+}$ concentrations.

\section{Discussion}

\section{Affinity and voltage dependence of the $\mathrm{Ca}^{2+}$ block}

The concentrations for half-maximal block at $V=0$ and the electrical distances reported here for block by external $\mathrm{Ca}^{2+}$ ions for the different channel types (see Table 2) are of the same order of magnitude as for other mammalian $\mathrm{Na}^{+}$channels. Nilius (1988), for example, determined $K_{d}(0)=35.1 \mathrm{~m} M$ and $\delta=0.36$ for heart muscle cells. However, the affinity for $\mathrm{Ca}^{2+}$ is much lower in squid axons, where a $K_{d}(0)$ of approximately $130 \mathrm{~m} M$ has been reported (Tanguy and Yeh 1988). This may represent adaptation of sea water animals to a higher $[\mathrm{Ca}]_{0}$. 


\section{Conclusions on structure-function relationships}

A model of the transmembrane topology of the different segments of the $\mathrm{Na}^{+}$channel sequence defined mainly by hydropathy has been proposed by Noda et al. (1986). More detailed models have been worked out by Catterall (1986) and Guy (1988). In the models, the S4 segments which are implicated in the voltage-dependent activation of $\mathrm{Na}^{+}$channels (Stühmer et al. 1989) traverse the membrane as $\alpha$-helices with the $\mathrm{N}$-terminal side being near the extracellular side of the membrane. According to the transmembrane topologies, homologous position 226, which carries the positive amino acid lysine $(\mathrm{K})$ in wildtype II channels and which is neutralized in the mutant $\mathrm{K} 226 \mathrm{Q}$, is located closer to the intracellular than the extracellular side of the membrane.

The results presented here show that the mutation K226Q changes the sensitivity of the $\mathrm{Na}^{+}$channel to extracellular $\mathrm{Ca}^{2+}$. This is manifested both by the voltage-dependent open-channel block and by the shift in steady-state activation. In contrast, the sensitivity to intracellular $\mathrm{Mg}^{2+}$ in the mutant differs little from that of wild-type II. At $30 \mathrm{mM}[\mathrm{Na}]_{i}$, the parameters describing the block by intracellular $\mathbf{M g}^{2+}$ are virtually identical (see Table 1). Affinities for $\mathrm{Mg}^{2+}$ differ at higher $[\mathrm{Na}]_{i}$ but this can be explained in terms of a multi-ion pore model (Begenisich and Cahalan 1980 a, b; Pusch 1990) by assuming slightly different values of the well depth representing the external binding site for $\mathrm{Na}^{+}$(unpublished calculation). The differences in the properties of the open-channel block by external $\mathrm{Ca}^{2+}$ are much more pronounced. The apparent affinities for $\mathrm{Ca}^{2+}$ (at $V=0$ ) are equal; however, the Hill coefficients needed to describe the data as well as the apparent electrical distances are significantly different (see Table 2). The $\mathrm{Ca}^{2+}$ blocking properties for the type II channel can be described by a Hill coefficient of about 0.5 . This indicates strong negative cooperativity, possibly due to another binding site for $\mathrm{Ca}^{2+}$ located near the channel opening. In contrast, the $\mathrm{Ca}^{2+}$ block for K226Q channels can be described by a Hill coefficient closer to 1 .

The existence of additional $\mathrm{Ca}^{2+}$ binding site(s) is also suggested by the shift of steady-state activation produced by external $\mathrm{Ca}^{2+}$. The larger effect observed for K226Q channels supports the idea that K226Q channels differ from the wild-type in a region of the channel protein which is located closer to the extracellular than to the intracellular side of the membrane.

Because the difference between wild-type and K226Q channels consists of a difference in charge it is tempting to explain the differences in $\mathrm{Ca}^{2+}$ sensitivity by surface charge effects. However, surface charge effects critically depend on local molecular configurations (Dani 1986). Since detailed structural information of the conformation of the $\mathrm{Na}^{+}$channel is lacking, any model which explains the Hill coefficient, introduced to account for the concentration dependence of the open-channel $\mathrm{Ca}^{2+}$ block, remains speculative. Shifts in activation produced by external $\mathrm{Ca}^{2+}$ could also be explained by different combinations of unspecific surface-charge screening and specific $\mathrm{Ca}^{2+}$ binding sites (Hille et al. 1975).
A simple interpretation of the results, as described above, would postulate that the mutation K226Q does not cause large conformational changes of the $\mathrm{Na}^{+}$channel protein. With this assumption it seems likely that position 226 is located close to the extracellular side of the membrane, in contrast to recent models of transmembrane topology of the $\mathrm{Na}^{+}$channel, in which position 226 of the S4 segment is close to the intracellular side of the membrane (Noda et al. 1986; Guy 1988).

As an alternative explanation, which is in agreement with these models, it could be imagined that the mutation K226Q causes the S4 segment of repeat I to be located, as a whole, closer to the intracellular side of the membrane than in the wild-type. Negative charges near the extracellular side, which are screened by the $\mathrm{N}$-terminal end of the S4 segment in the wild-type, might be less screened in K226Q channels. This localized increase in net negative charge could account for the differences in sensitivity to external $\mathrm{Ca}^{2+}$. In this case, conformational rearrangements of the protein segment close to the intracellular side of the membrane would also be expected. However, large conformational changes are not consistent with the results obtained for the $\mathrm{Mg}^{2+}$ block. In addition, the similarities between the mutant and the wild-type with respect to single-channel conductance and inactivation (Stühmer et al. 1989) argue against large conformational changes. Lacking additional structural information, the simpler interpretation that position 226 is located close to the extracellular side of the membrane seems more plausible.

A dynamic model of the action of external $\mathrm{Zn}^{2+}$ on gating kinetics of squid axon $\mathrm{Na}^{+}$channels has been proposed by Gilly and Armstrong (1982). In their model, $\mathrm{Zn}^{2+}$ binds to the channels in its closed state at a negatively charged site which then moves inward when the channel opens. By analogy, it is possible that neutralization of the positive lysine at position 226 increases the affinity for $\mathrm{Ca}^{2+}$ of this binding site in the closed state and thereby increases the effects of $\mathrm{Ca}^{2+}$ on steady-state activation. With this assumption, position 226 would be most likely located close to the extracellular side in the closed state of the channel. Alternatively, the negative site binding $\mathrm{Zn}^{2+}$ (or $\mathrm{Ca}^{2+}$ ) in the closed state, instead of moving inwards, could be screened by the approach of a positive amino acid, like K226, upon activation. In this case, position 226 might be located close to the extracellular side of the membrane in the open state. The latter interpretation is consistent with recent models of $\mathrm{Na}^{+}$channel gating (Guy and Conti 1990).

The present study is the first attempt to use divalentcation effects as a tool to investigate structure-function relationships of cloned $\mathrm{Na}^{+}$channels, yielding important information concerning the topology of the $\mathrm{S} 4$ segments. Application of this approach to different mutants, including ones with neutralized negative charges, will increase the understanding of the topology and the dynamics of the $\mathrm{Na}^{+}$channel protein.

Acknowledgements. I thank M. Papke for help with the injection of oocytes and expert technical assistance, M. Pilot for the preparation of chromaffin-cells, G. Soff for a program solving differential 
equations, Drs. W. Stühmer, M. D. Cahalan, E. Neher, R. Davis, and F. Conti for critical comments on the manuscript and Prof. S. Numa, Dr. M. Noda and their collaborators in Kyoto for supplying mRNA. This study was supported in part by EEC grant $\mathrm{SC} 1 * 305$.

\section{References}

Begenisich TB, Cahalan MD (1980a) Sodium channel permeation in squid axons I: reversal potential measurements. J Physiol (London) 307:217-242

Begenisich TB, Cahalan MD (1980 b) Sodium channel permeation in squid axons II: non-independence and current-voltage relations. J Physiol (London) 307:243-257

Catterall WA (1986) Molecular properties of voltage-sensitive sodium channels. Ann Rev Biochem 55:953-958

Dani JA (1986) Ion-channel entrances influence permeation. Net charge, size, shape, and binding considerations. Biophys J 49:607-618

Frankenhaeuser B, Hodgkin AL (1957) The action of calcium on the electrical properties of squid axons. J Physiol (London) $137: 218-244$

Gilly WmF, Armstrong CM (1982) Slowing of sodium channel opening kinetics in squid axon by extracellular zinc. J Gen Physiol 79:935-964

Guy HR (1988) A model relating the structure of the sodium channel to its function. In: Agnew WS, Claudio T, Sigworth FJ (eds) Molecular biology of ionic channels. Current Topics in Membranes and Transport, vol 33. Academic Press, San Diego, pp 289-308

Guy HR, Conti F (1990) The propagating helix model of voltagegated channeis. Biophys J 57:111 a

Hahin R, Campbell DT (1983) Simple shifts in the voltage dependence of sodium channel gating caused by divalent cations. $\mathbf{J}$ Gen Physiol 82:785-805
Hille B, Woodhull AM, Shapiro BI (1975) Negative surface charge near sodium channels of nerve: divalent ions, monovalent ions, and $\mathrm{pH}$. Phil Trans R Soc Lond B 270:301-318

Hodgkin AL, Huxley AF (1952) A quantitative description of membrane current and its application to conduction and excitation in nerve. J Physiol (London) 117:500-544

Kayano T, Noda M, Flockerzi V, Takahashi H, Numa S (1988) Primary structure of rat brain sodium channel III deduced from the cDNA sequence. FEBS Lett 228:187-194

Noda M, Ikeda T, Kayano T, Suzuki H, Takeshima H, Kurasaki M, Takahashi H, Numa S (1986) Existence of distinct sodium channel messenger RNAs in rat brain. Nature (London) 320:188-192

Nilius B (1988) Calcium block of guinea-pig heart sodium channels with and without modification by the piperazinylindole DPI 201-106. J Physiol (London) 399:537-558

Pusch M (1990) Open-channel block of $\mathrm{Na}^{+}$channels by intracellular $\mathrm{Mg}^{2+}$. Eur Biophys J 18:317-326

Stühmer W, Methfessel C, Sakmann B, Noda M, Numa S (1987) Patch clamp characterization of sodium channels expressed from rat brain cDNA. Eur Biophys J 14:131-138

Stühmer W, Conti F, Suzuki H, Wang X, Noda M, Yahagi N, Kubo $H$, Numa S (1989) Structural parts involved in activation and inactivation of the sodium channel. Nature (London) 339:597603

Tanguy J, Yeh JZ (1988) Divalent cation block of normal and BTXmodified sodium channels in squid axons. Biophys $\mathrm{J}$ 53:229 a

Taylor RE, Armstrong CM, Bezanilla F (1976) Block of sodium channels by external calcium ions. Biophys $\mathcal{J} 16: 27$ a

Vandenberg CA, Bezanilla F (1988) Single-channel, macroscopic and gating currents from $\mathrm{Na}$ channels in squid giant axon. Biophys J 53:226a

Woodhull AM (1973) Ionic blockage of sodium channels in nerve. J Gen Physiol 61:687-708

Worley III JF, French RJ, Krueger BK (1986) Trimethyloxonium modification of single batrachotoxin-activated channels in planar lipid bilayers. J Gen Physiol 87:327-349

Yamamoto D, Yeh JZ, Narahashi T (1984) Voltage-dependent block of normal and tetramethrin-modified single sodium channels. Biophys J 45:337-344 